\title{
Genetic characterization of psp encoding the DING protein in Pseudomonas fluorescens SBW25 Xue-Xian Zhang*1,2, Ken Scott ${ }^{1}$, Rebecca Meffin ${ }^{1}$ and Paul B Rainey ${ }^{1,2}$
}

Address: 1School of Biological Sciences, University of Auckland, Private Bag 92019, Auckland, New Zealand and 2Institute of Molecular Biosciences

Email: Xue-Xian Zhang* - x.x.zhang1@massey.ac.nz; Ken Scott - k.scott@auckland.ac.nz; Rebecca Meffin - rmef001@ec.auckland.ac.nz; Paul B Rainey - p.b.rainey@massey.ac.nz

* Corresponding author

Published: 18 December 2007

BMC Microbiology 2007, 7:114

This article is available from: http://www.biomedcentral.com/I47/-2/80/7/II4

(C) 2007 Zhang et al; licensee BioMed Central Ltd.

This is an Open Access article distributed under the terms of the Creative Commons Attribution License (http://creativecommons.org/licenses/by/2.0), which permits unrestricted use, distribution, and reproduction in any medium, provided the original work is properly cited.
Received: 17 April 2007

Accepted: 18 December 2007

\begin{abstract}
Background: DING proteins constitute a conserved and broadly distributed set of proteins found in bacteria, fungi, plants and animals (including humans). Characterization of DING proteins from animal and plant tissues indicated ligand-binding ability suggesting a role for DING proteins in cell signaling and biomineralization. Surprisingly, the genes encoding DING proteins in eukaryotes have not been identified in the eukaryotic genome or EST databases. Recent discovery of a DING homologue (named Psp here) in the genome of Pseudomonas fluorescens SBW25 provided a unique opportunity to investigate the physiological roles of DING proteins. P. fluorescens SBW25 is a model bacterium that can efficiently colonize plant surfaces and enhance plant health. In this report we genetically characterize Psp with a focus on conditions under which psp is expressed and the protein exported.
\end{abstract}

Results: Psp is closely related to the periplasmic $P_{i}$ binding component of the $A B C$-type phosphate transporter system (Pst). psp is flanked by a gene cluster predicted to function as a type II protein secretion system $(\mathrm{Hxc})$. Deletion analysis combined with chromosomally integrated 'lac Z fusions showed that both $p s p$ and $p s t C$ are induced by $P_{i}$ limitation and that $p s t C$ is required for competitive growth of the bacterium in $P_{i}$ limited medium. $h x c R$ is not regulated by $P_{i}$ limitation. Psp was detected (using anti-DING serum) in the supernatant of wild-type culture but was greatly reduced in the supernatant of an isogenic strain carrying an $h x c R$ mutation $(\Delta h x c R)$. A promoter fusion between $h x c R$ and a promoterless copy of a gene ('dapB) essential for growth in the plant environment showed that expression of $h x c R$ is elevated during colonization of sugar beet seedlings. A similar analysis of psp showed that it is not induced in the plant environment.

Conclusion: Psp gene is expressed under conditions of $\mathrm{P}_{\mathrm{i}}$ limitation. It is an exoprotein secreted mainly via the Hxc type II secretion system, whose expression is elevated on plant surfaces. We propose that Psp is involved in extracellular scavenging of phosphates, which are subsequently taken up by the cell-bound Pst transport system.

\section{Background}

DING proteins, named for the highly-conserved N-termi- nal sequence (DINGGG-), were initially described as proteins from animal and plant tissues with molecular 
weights about $38-40 \mathrm{kDa}$. The first DING protein was identified from human rheumatoid arthritis (RA) synovial fluid as a lymphocyte stimulatory protein [1]. Since then human DING proteins have been identified independently from urine and kidney stones as crystal adhesion inhibitors [2]; in skin fibroblasts and cervical carcinoma cells using hirudin-agarose affinity columns [3]; and in breast cells by their high affinity for genistein, an estrogen analog [4]. In these latter two cases, extracellular DING protein is linked to cell growth promotion by an autocrine or paracrine mechanism [3,4]. DING proteins have also been reported from other animals, e.g., turkey (as a lipid-free polysaccharide-binding protein) and rat (as a cotinine receptor). DING proteins from several plant and fungal species are characterized by short N-terminal sequences and one has been shown capable of binding a germin-like protein in tobacco [5]. Together these studies suggested that DING proteins are widespread in eukaryotes and play important roles in cell signaling and biomineralization.

Although DING proteins have been frequently isolated or identified from eukaryotes on the basis of ligand-binding properties, no complete gene or protein sequences have been found in either the current eukaryotic genomes or EST databases. Recently, Morales et al. [6] reported the first complete amino acid sequence of a DING protein isolated from the human plasma high-density lipoprotein fraction, though no corresponding gene sequence has been identified. This DING protein was structurally characterized to be a phosphate binding protein, which is functionally related to solute binding proteins of the Pst systems, the bacterial ABC (ATP-binding cassette) transporters specific for phosphate [6]. The Pst system is comprised of four components [7]: a periplasmic phosphate binding protein (PstS), two integral membrane domains (PstC and PstA) and a cytoplasmic ATPase (PstB).

Low-level homology between eukaryotic DING proteins and bacterial phosphate-binding proteins has been known for some time [5]. More recently, proteins with a greater degree of homology have been predicted in the sequenced genomes of Pseudomonas [8], including P. fluorescens SBW25 [9]. P. fluorescens SBW25 is a Gram-negative plant growth-promoting rhizobacterium (PGPR) representative of a group of fluorescent pseudomonads isolated from the field-grown sugar beets at the University of Oxford farm, Wytham, Oxford, in 1989 [10]. The DING homologue of $P$. fluorescens SBW25, named Psp here for phosphate scavenging protein, shows $65-75 \%$ identity with the partial eukaryotic DING protein sequences and contains the highly conserved phosphate-binding site [9]. However, prediction of flanking genes in the $p s p$ locus was inaccurate when the analysis was based on raw SBW25 shotgun genome sequence data available at that time [9].
The coding region of $p s p$ of $P$. fluorescens SBW25 was previously cloned and expressed in Escherichia coli and subsequent phosphate binding assays showed that the expressed SBW25 DING protein is able to bind inorganic phosphate $\left(\mathrm{P}_{\mathrm{i}}\right)$ in vitro [9]. Secondary and tertiary structure prediction and determination indicated a PstS-like, "Venus flytrap" structure $[9,11,12]$. A similar structure has been found for the human plasma phosphate binding protein [6]. Previous study also showed that the DING protein was not detectable in SBW25 cells grown in LuriaBertani (LB) medium [9], suggesting that $p s p$ was not constitutively expressed.

In this report we describe the genetic characterization of $p s p$ (pflu2427) encoding the DING protein of $P$. fluorescens SBW25. We focus on the conditions under which psp is expressed and its role for bacterial growth in laboratory media and in the plant environment. Our work begins with the complete but un-annotated whole genome sequence of SBW25. Based initially on this sequence, we perform an up-to-date comparison of gene sequences of the phosphate binding proteins in SBW25 and other genome-sequenced Pseudomonas. We examine the functionalities of $p s p$, together with the genes located upstream of $p s p$ ( $h x c R S$ ) and genes in the pst locus, using a combination of site-directed mutagenesis and chromosomally integrated 'lacZ fusions. The role of the DING protein in bacterial phosphate metabolism and plant colonization is discussed.

\section{Results \\ Genomic analyses of putative phosphate-binding proteins in Pseudomonas}

The genome of $P$. fluorescens SBW25 harbors two putative phosphate-binding proteins, Psp (Pflu2427) and PstS (Pflu3318), which show 38\% amino acid sequence similarity to each other. Both Psp and PstS possess the highly conserved phosphate-binding sites [5]. However, PstS is 52 amino acids shorter than Psp and it does not contain the N-terminal DINGGG residue.

$p s t S$ is the first of six genes which are organized in the same orientation, suggesting that they are co-transcribed as an operon (Figure 1A). Genes encoding the other three components of the Pst system (PstCAB) are located downstream of $p s t S$. The putative pst genes are organized by a gene order typical for $\mathrm{ABC}$ transporters involved in substrate uptake: the periplasmic binding protein (PstS) first and then the membrane-spanning domains (PstC and PstA) and the ATP-binding cassette (PstB).

$p s p$ is located $1035 \mathrm{~Kb}$ away from pstS. ORF pflu2428, downstream of $p s p$, encodes a predicted protein of 4083 amino acids, which contains a haemagglutination activity domain (Figure 1B). This domain is typical of haemagglu- 
A

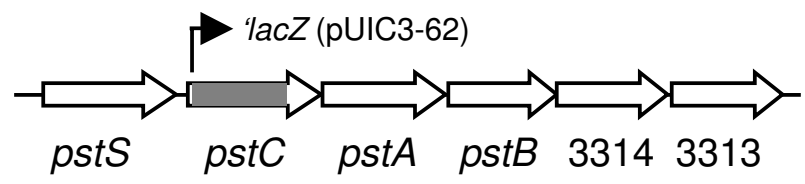

B
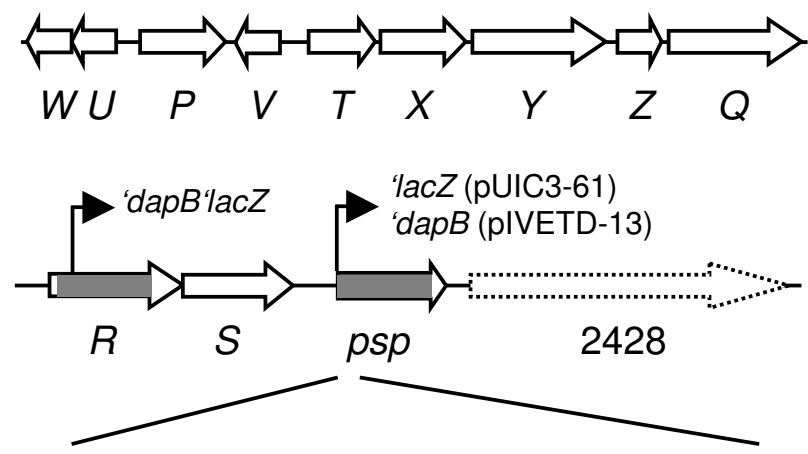

MFKRNVLAVSMTLAALCSAQAAMADINGGGATL个

C

CACGTTCATAAAACCGTAACAAGATTGTTGCAG AGTTGCCGAGCCCGAAATCACAAGGATTTTCCT TAATGAA - pstS

GCTGTCATCGGAAGCTGCGAAATTTCCTACCC ATGGCCTCACCCCTCGCCACCCCCGCAAACCG GCTTGGGACCGAAGCCATGGCGTCATGCAAGA GCCATCTACCCCAACGTACAAACACGACGAAㅁ GGAGATTCTTCATGTT - psp

\section{Figure I}

Genetic organization of the pst (A) and psp (B) loci in $\boldsymbol{P}$. fluorescens SBW25. Regions deleted in mutants PBR827 ( $\Delta$ pstC or $\triangle$ pflu33 I 7), PBR828 ( $\Delta h x c R$ or $\Delta$ pflu2424) and PBR826 ( $\Delta p s p$ or $\Delta p$ flu2427) are marked by grey. Positions of the promoterless 'lac $Z$ and 'dapB fusions are shown by arrowed lines. The predicted signal peptide cleavage site is indicated by vertical arrow. ORF Pflu2428 is not drawn to scale and shown by discontinued arrow bar. (C) Promoter regions of pst and psp operons. The putative translational start and ribosome-binding sites are indicated by bold type and underlined letters, respectively. The predicted Pho box sequences are boxed and nucleotides identified in the $E$. coli Pho box consensus [CTGT-

CATA(AT)A(TA)CTGT(CA)A(CT)] [7] are highlighted by bold type. tinins, which are involved in bacterial adhesion to host cells [13]. Immediately upstream of $p s p$ is a cluster of 11 genes that are predicted to encode an alternative type II secretion system (Hxc) [14]. Type II-dependent exoproteins have an $\mathrm{N}$-terminal signal peptide that is cleaved off during translocation to the periplasm by the Sec or Tat export system [15]. The folded or mature substrate protein is subsequently transported across the outer membrane by the type II secretion apparatus. The discovery of $h x c$ flanking $p s p$ led to a signal peptide analysis of the deduced amino acid sequence of $p s p$ by using the SignalP 3.0 Server [16]. Results showed that Psp does contain a signal peptide and the predicted cleavage site just before the characteristic DINGGG residue (Figure 1B). The fact that a type II secretion system is present in the psp locus and that Psp has a signal peptide led us to predict that Psp is an extracellular protein secreted by the Hxc system. This hypothesis was experimentally tested and described below.

Comparative genomic analysis of putative phosphatebinding proteins between $P$. fluorescens SBW25 and other genome-sequenced Pseudomonas showed that PstS is ubiquitous whereas the DING protein is only present in two other species or strains (P. fluorescens Pf- 5 and $P$. aeruginosa PA14). P. aeruginosa PAO1 contains two DING-like proteins (LapA and LapB), of which the N-termini (VTGGG) show partial similarity to the DINGGG residues. The lap $A B$ genes were previously shown to encode lowmolecular-weight alkaline phosphatases [14,17]. A phylogenetic tree based on the deduced amino acid sequences is shown in Figure 2. Clearly, the DING proteins form a separate group from the DING-like (LAP) and the PstS proteins, as already reported [8], but they are much more diverse than previously anticipated.

\section{The psp and pst operons are induced by $\boldsymbol{P}_{\boldsymbol{i}}$ limitation}

To determine the conditions under which Psp is expressed, a transcriptional 'lacZ fusion was constructed to $p s p$ and the resulting fusion plasmid (pUIC3-61) was integrated into the genome of SBW25 by a single event of homologous recombination. The resulting $p s p$-'lac $Z$ fusion strain (PBR829) was subjected to a $\beta$-galactosidase assay using cells grown in both phosphate-rich (PR) and phosphate-limited (PL) medium. Results (Table 1) showed that $p s p$ expression was elevated 3.85 -fold at 4 hours and 64.72-fold at 26 hours, in cells grown in PL compared to cells grown in PR medium.

Previous studies on transcription of pst genes from $P$. aeruginosa PAO1 and E. coli showed that the pst operon is expressed under $\mathrm{P}_{\mathrm{i}}$ limitation $[7,18]$. To test whether the predicted pst operon of SBW25 is induced by $\mathrm{P}_{\mathrm{i}}$ limitation, a pstC-'lacZ fusion strain (PBR830) was constructed and its expression was tested in a similar way as the $p s p-' l a c Z$ fusion strain. Results (Table 1) showed that pstC expres- 


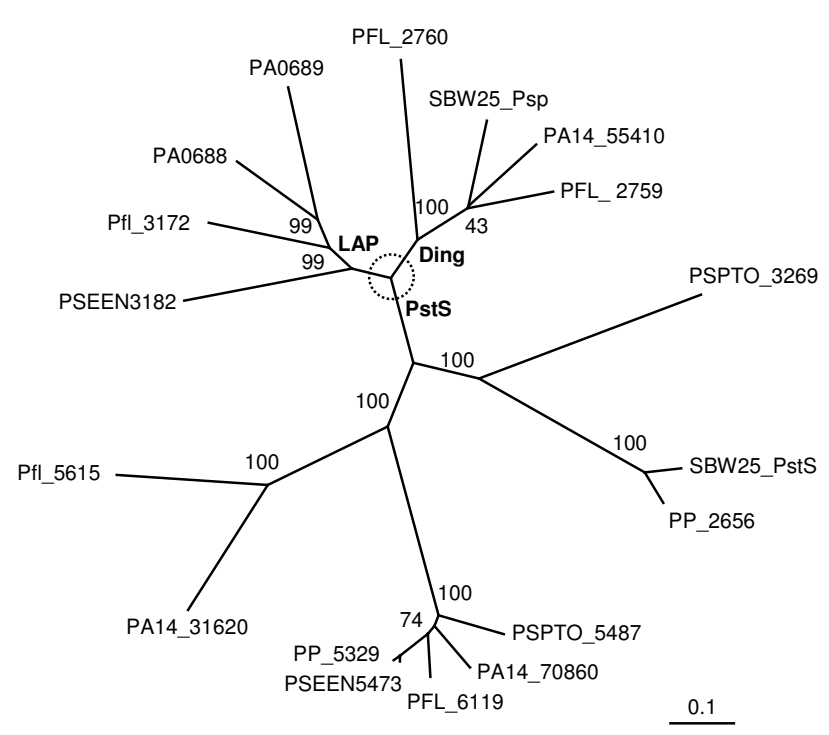

Figure 2

The neighbor-joining tree showing phylogenetic relationships of putative phosphate-binding proteins from Pseudomonas. The deduced amino acid sequences are derived from the Pseudomonas genome databases: $P$. aeruginosa PAI4 (PAI4_554I0, PAI4_70860 and PAI4_3 I620); P. aeruginosa PAOI [PA0688 (LapA), PA0689 (LapB) and PA5369 (it is identical to PAI4_70860 thus not shown in the tree); P. putida KT2440 (PP_5329, PP_2656); P. fluorescens Pf5 (PFL_2759, PFL_2760 and PFL_6I I9); P. fluorescens PfO-I (Pfl_3I72 and Pfl_56I5); P. syringae pv. tomato DC3000 (PSPTO_3269 and PSPTO_5487); P. entomophila L48 (PSEEN3 I 82 and PSEEN5473). Percentage bootstrap values obtained from 1000 trials are shown on branches. The scale bar refers to the number of substitutions per site.

sion was significantly higher for cells grown in PL medium compared with cells grown in PR medium. Compared to the levels of $p s p$ expression, the basic $p s t C$ expression and $\mathrm{P}_{\mathrm{i}}$ limitation-induced expression are very low (Table 1).

Table I: Expression of the Psp and Pst genes in P. fluorescens SBW25

\begin{tabular}{llll}
\hline lacZ fusion & Medium & \multicolumn{2}{c}{$\beta$-galactosidase $(\mathrm{amol} 4 \mathrm{MU} / \mathrm{min} / \mathrm{cell})^{\mathrm{a}}$} \\
\cline { 3 - 4 } & & 4 hours & 26 hours \\
\hline \multirow{2}{*}{ psp-'lacZ } & PR & $0.22 \pm 0.03$ & $0.22 \pm 0.07$ \\
& PL & $0.83 \pm 0.35(3.85)$ & $14.47 \pm 3.53(64.72)$ \\
pstC-'lacZ & PR & $0.1 \pm 0.01$ & $0.16 \pm 0.02$ \\
& PL & $0.14 \pm 0.02(1.48)$ & $0.58 \pm 0.06(3.61)$
\end{tabular}

a Fold of increase for cells grown in PL medium versus PR medium is shown in parenthesis.
Next we carefully examined the promoter regions of $p s p$ and pst to search for putative Pho box sequences. As shown in Figure 1C, sequence motifs similar to the E. coli Pho box consensus were identified upstream of the translational start site of $p s t S$ and $p s p$, suggesting that $p s p$ and $p s t$ operons are subjected to control by the conserved Pho regulon $[7,19]$.

\section{Mutagenesis analysis of psp and pstC}

Having demonstrated that $p s p$ and $p s t C$ are induced by $\mathrm{P}_{\mathrm{i}}$ limitation, we next asked whether they are required for bacteria to grow in laboratory medium with limited $\mathrm{P}_{\mathrm{i}}$ and in the plant environment. To do this, two in-frame deletion mutants PBR826 ( $\Delta p s p)$ and PBR827 ( $\Delta p s t C)$ were constructed by a standard procedure of allelic exchange. The two mutant strains showed similar growth curves with the wild-type when growing in complete medium (LB), minimal medium (M9) and phosphate media PR and PL (data not shown).

To assess the growth more rigorously, we determined the competitive ability of each mutant relative to the wild type ancestor in two laboratory media (PR and PL). The mutant strain was mixed 1:1 with a lacZ-marked "wild type" strain (SBW25-lacZ) and the bacterial mixture was inoculated into the tested media. After growing in competition for $\sim 20$ generations in the laboratory media, the ratio of the mutant to the wild type competitor was determined on LB plus X-gal agar plates. Results are shown in Figure 3. Growth of PBR827 $(\Delta p s t C)$ was comparable to the wild-type in $\mathrm{PR}$ medium but was significantly impaired in PL medium. No fitness effect was observed with PBR826 ( $\Delta p s p)$.

The competitive ability of each mutant ( $\Delta p s p$ and $\Delta p s t C$ ) relative to the lacZ-marked "wild type" strain was also determined during a course of colonization of sugar beet seedlings. Each mutant strain was inoculated, with the wild type in a 1:1 ratio, onto sugar beet seeds and recovered in two weeks from the shoots and rhizosphere on M9 plates supplemented with X-gal. Results showed that the fitness of PBR826 $(\Delta p s p)$ and PBR827 $(\Delta p s t C)$ was not significantly impaired in either the shoots or the rhizosphere (data not shown).

\section{Psp is secreted by the Hxc system}

Armed with knowledge that $p s p$ is expressed in $P_{\mathrm{i}}$ limited medium, we next asked whether Psp is an extracellular protein and furthermore whether it is secreted via the Hxc system. An immunodiffusion experiment was performed between anti-DING antiserum and concentrated supernatants of SBW25 (wild-type) cultures in PL and also in PR and LB broths (as controls). Results showed that a precipitin line was formed only between anti-DING serum and PL supernatant (data not shown). From this experiment 


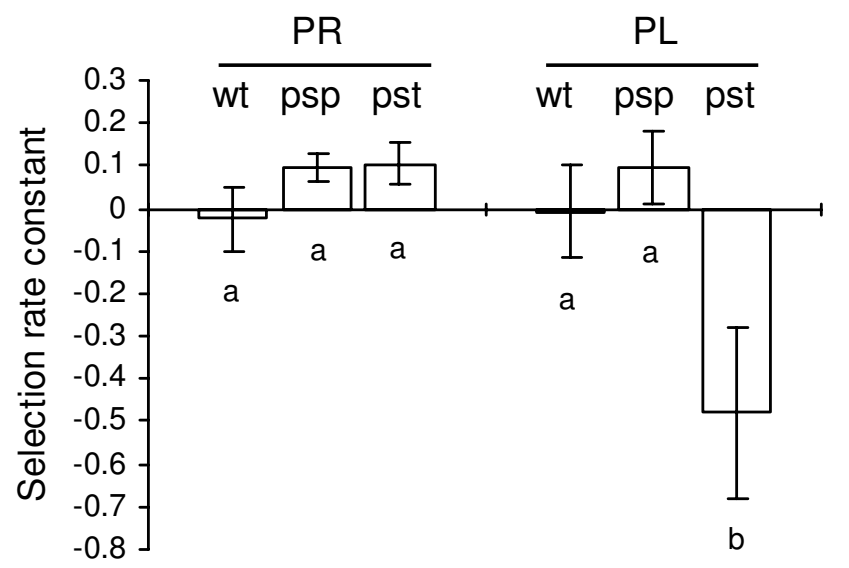

Figure 3

Fitness of $P$. fluorescens SBW25 and derived mutants. Fitness of SBW25 (wt) and mutants PBR826 ( $\Delta$ psp, psp) and PBR827 ( $\triangle$ pstC, pst) relative to SBW25-lacZ was measured with cells grown in $\mathrm{P}_{\mathrm{i}}$-rich $(\mathrm{PR})$ and $\mathrm{P}_{\mathrm{i}}$-limited $(\mathrm{PL})$ media. Data are means and standard errors of $\sim 8$ independent cultures. A fitness of zero indicates that the fitness of the mutant is identical to wild type (a negative value indicates a reduction in fitness relative to wild type). Bars identified by different letters are significantly different $(P<0.05)$ by Tukey's HSD.

we conclude that Psp is an exoprotein, and its expression is induced by $P_{i}$ limitation, which is consistent with the $\beta$ galactosidase activity data described above and is further confirmed by the Western blotting analysis described below.

To test whether Psp secretion is dependent on the Hxc system, an in-frame $h x c R$ (pflu2424) deletion mutant was constructed by allelic exchange. $h x c R$ encodes an essential component of the type II secretion system (ATPase), which provides energy for substrate translocation. Concentrated supernatants and cell lysates of the mutant strain PBR828 $(\Delta h x c R)$ grown in PL medium were subjected to immunodiffusion analysis with an anti-DING antibody. Wild-type SBW25 and PBR826 ( $\Delta p s p)$ were included and tested in parallel as the positive and negative control, respectively. Figure 4A shows that Psp is present in both supernatant and lysate of wild-type cells, but is not present in the lysate or supernatant from PBR826 $(\Delta p s p)$. In PBR828 $(\Delta h x c R)$, Psp is detectable at normal concentrations in the lysate, but is present at much lower concentrations in the supernatant, indicating that secretion is significantly reduced by the $\Delta h x c R$ mutation.

This result was confirmed by Western blot analysis of the same bacterial supernatants: only in PL medium was a 40 kDa protein detectable with anti-DING antibodies (Figure
4B). Figure 4C showed that DING was detected as a strong band in the supernatant of the wild-type culture, but was totally absent in mutant PBR826 ( $\Delta p s p)$, and greatly reduced $(<15 \%)$ in the PBR828 $(\Delta h x c R)$ supernatant. This Western blot result indicates that the Psp secretion is predominantly via the Hxc system.

\section{HxcR is not induced by $P_{i}$ limitation but shows elevated level of expression in planta}

The in vivo expression technology (IVET) has been employed to identify SBW25 genes showing elevated levels of expression on the plant surfaces $[20,21]$. These plant inducible genes are of particular interest because they are predicted to play a role in the maximization of ecological performance in planta - a prediction confirmed for a locus encoding an acetylated cellulose polymer [20] and cueA, a gene encoding the copper-transporting P-type ATPase [22].

Central to this technique is a $d a p B$ deletion mutant strain (SBW25 $\Delta d a p B$ ), which is unable to grow in the absence of an exogenous source of DAP (diaminopimelic acid) and lysine and, as a result, is unable to colonize sugar beet seedlings [20]. By fusing random fragments of the SBW25 genome to a promoterless copy of $d a p B$ it is possible to identify plant-inducible loci. Arising from a genome-wide screen for plant-inducible loci from SBW25 was strain PIL082, which contains a fusion between $h x c R$ and 'dapB (Figure 1B) suggesting that the $h x c$ locus is plant-induced.

Experimental evidence described above showed that $p s p$ is induced by $\mathrm{P}_{\mathrm{i}}$ limitation, but the precise location of the $p s p$ promoter was unclear. $h x c R$ and $p s p$ are organized in the same orientation. Thus it is possible that $p s p$ is cotranscribed with $h x c R S$ and induced also in the plant environment. To test this hypothesis, an IVET fusion ( $p s p$-'dapB) was constructed by cloning an 800 nt DNA fragment from $h x c S$ to $p s p$ into pIVETD, and integrated into the genome by homogenous recombination. The resultant IVET fusion (PIL082-1) was subjected to a plant colonization assay, together with the $h x c R$ IVET fusion strain (PIL082). About 1000 cells were inoculated onto each sugar beet seed and bacteria were recovered from the shoots and rhizosphere, two weeks after plant sowing. Results showed that the psp IVET fusion strain was not detected (under the detection limit of 30 cells per shoot, 300 cells per rhizosphere), whereas the $h x c R$ fusion strains reach a cell density of $6.7 \pm 3.4 \times 10^{2}$ cells per shoot and $5.46 \pm$ $2.30 \times 10^{4}$ per rhizosphere. Both PIL082 and PIL082-1 are unable to grow in minimal medium (M9) in the absence of lysine and DAP. The ability of PIL082 to grow on sugar beet seedlings but not on M9 medium confirmed that the promoter driving $h x c R$ expression is active in the plant environment. The inability of PIL082-1 to grow in planta indicates that $p s p$ is not induced in the plant environment. 

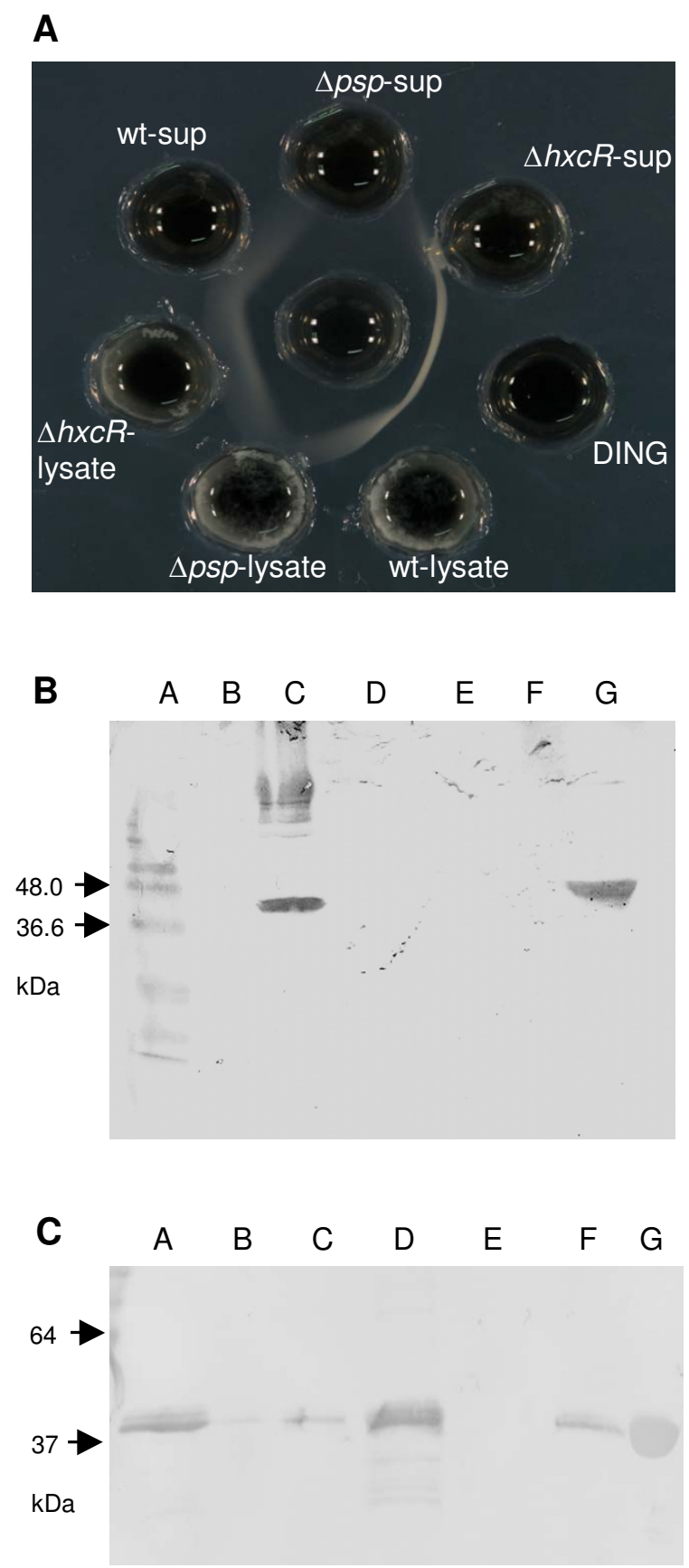

\section{Figure 4}

Immunochemical analysis of Psp in P. fluorescens SBW25 and derived mutants. (A) Double immunodiffusion of cell supernatants and lysates. The central well in a $1.5 \%$ agarose plate contained rabbit anti-DING (Psp from SBW25) antiserum. The peripheral wells contained (clockwise, from the top) PBR826 ( $\Delta p s p)$ supernatant ( $\Delta p s p$-sup), PBR828 ( $\Delta h x c R)$ supernatant ( $\Delta h x c R$-sup), Psp standard (DING), SBW25 lysate (wt-lysate), PBR826 ( $\Delta p s p)$ lysate ( $\Delta p s p$-lysate), PBR828 ( $\Delta h x c R)$ lysate ( $\Delta h x c R$-lysate), SBW25 supernatant (wt-sup). (B) Western blotting of supernatants for SBW25 grown in different media. Concentrated cell supernatants were separated on a I2\% SDS-PAGE gel, and Psp was detected with anti-human DING antiserum. Lane A, protein ladder; B, PBR826 ( $\triangle p s p)$ in LB broth (negative control); C, SBW25 in PL broth; D, E, SBW25 in PR broth; F, SBW25 in LB broth; G, recombinant Psp (positive control). (C) Western blotting of cell supernatants for SBW25 mutants grown on PL medium. Concentrated cell fractions were separated on a $12 \%$ SDS-PAGE gel, and Psp was detected with anti-Psp antiserum. Lane A and D, wild-type; B and E, PBR826 ( $\Delta p s p) ; \mathrm{C}$ and F, PBR828 ( $\Delta h x c R)$; G, Psp standard. 
To test whether $h x c R$ is induced by $\mathrm{P}_{\mathrm{i}}$ limitation, expression of PIL082 fusion was examined by measuring the $\beta$ galactosidase activities in cells grown in PR and PL media (the IVET vector pIVETD contains a second marker gene 'lacZ located downstream of 'dapB) [20]. Results showed that $h x c R$ expression was not elevated in cells grown in PL medium $(0.84 \pm 0.06 \mathrm{amol} 4 \mathrm{MU} / \mathrm{min} / \mathrm{cell})$ compared to cells grown in PR medium $(0.97 \pm 0.02 \mathrm{amol} 4 \mathrm{MU} / \mathrm{min} /$ cell). Taken together, the expression data (both in vitro and in vivo) indicate that $h x c R$ and $p s p$ are transcribed by different promoters. Given that $h x c R$ and $h x c S$ are organized in a contiguous manner, the $p s p$ promoter is likely to be located in the intergenic region of $h x c S$ and $p s p$ (Figure $1 \mathrm{~B})$. This is consistent with the tentative identification of a putative Pho box in this region (Figure 1C).

\section{Discussion}

Phosphate is an essential but often limiting nutrient. Multiple $P_{i}$ transport systems have evolved in bacteria to facilitate efficient uptake of $\mathrm{P}_{\mathrm{i}}$ from the environment. In E. coli, a constitutively-expressed, low-affinity transporter (Pit system) is used in cells grown in environments with excess $\mathrm{P}_{\mathrm{i}}$. Under conditions of $\mathrm{P}_{\mathrm{i}}$ limitation, a high-affinity transport system (Pst) is induced and operates for $\mathrm{P}_{\mathrm{i}}$ scavenging [7]. In this report, we identified by in silico analysis genes encoding the Pst transporter system in the SBW25 genome, whose function we experimentally tested. The Pst system is an ABC-type transporter that takes up $P_{i}$ from the periplasmic space of Gram negative bacteria. Psp, a protein secreted outside of the cell, may act to scavenge $P_{i}$ to the cell surface, where it can be subsequently taken up by the Pst system. Therefore, the Psp and Pst systems are very likely involved in phosphate acquisition in a cooperative manner. Although $p s p$ is induced by limiting phosphate concentrations, its absence does not limit bacterial competitive growth under laboratory conditions, unlike $p s t C$. The DING-like LAP proteins may hydrolyze environmental organophosphates, following secretion, which is also consistent with a phosphate-scavenging role. It is noteworthy that PstS sequences which are duplicated in some Pseudomonas genomes may be significantly different, implying that there may also be functional variance within this sub-family of phosphate-binding proteins.

Bacteria living in complex environments, e.g. plants and animals, utilize the type II secretion pathway for extracellular release of particular proteins, such as toxins and hydrolytic enzymes [15]. Two functionally independent type II secretion systems (Xcp and Hxc) have been described in $P$. aeruginosa [14]. Interestingly, in addition to the Xcp and Hxc homologues, the P. fluorescens SBW25 genome harbors an additional copy of the Type II gene cluster (Rainey lab, unpublished). Here we showed that the DING protein secretion was greatly reduced in the HxcR mutant PBR828 $(\Delta h x c R)$, suggesting that there may be some functional redundancy amongst the three type II secretion systems.

Psp is regulated by $P_{i}$ limitation but its expression is not elevated in the plant environment, indicating that $\mathrm{P}_{\mathrm{i}}$ is not depleted in our plant growth system. On the contrary, $h x c R$ is not induced by $\mathrm{P}_{\mathrm{i}}$ limitation but shows elevated levels of expression in sugar beet seedlings. While the result implicates important roles of the Hxc secretion system in bacterium-plant interaction, like most plant-inducible genes $[20,21]$, the signals that induce $h x c R$ expression in planta are currently unknown.

\section{Conclusion}

Proteins belonging to the DING family have very poorly defined physiological roles. Functional investigation of eukaryotic DING proteins is hindered by the unavailability of full amino acid sequences in most cases and by the absence of full gene sequences in the eukaryotic genome and EST databases. Insight into the function and gene regulation of the prokaryotic members of DING protein family may provide valuable cues as to the functions of the DING proteins in general. A previous study showed that Psp from $P$. fluorescens SBW25 is capable of binding $P_{\mathrm{i}}$ in vitro [9]. In this report we demonstrated in $P$. fluorescens SBW25 that Psp is expressed under $P_{\mathrm{i}}$ limitation and is an exoprotein, secretion of which is mainly dependent on the alternative type II secretion system (Hxc). Interestingly, expression of the Hxc system is elevated on plant surfaces, which is first reported here. Moreover, the Pst $P_{i}$ transporter system was predicted in the SBW25 genome and its function was experimentally characterized in parallel to Psp. Results show that Pst is induced by phosphate limitation and it is required for bacterial competitive growth in phosphate limiting medium. Taken together, the data implicate functional roles of Psp in bacterial phosphate metabolism. Psp and Pst could form an efficient phosphate scavenging system, which contributes to physiological adaptation to phosphate limited environments.

\section{Methods \\ Bacterial strains, plasmids and growth conditions}

The ancestral strain of $P$. fluorescens SBW25 is a plasmidfree, non-pathogenic bacterium belonging to the rRNA group I of fluorescent Pseudomonas isolated from sugar beet phytosphere [10]. Escherichia coli DH5aגpir [23] was used as a recipient strain for gene cloning and then a donor for conjugative transfer into Pseudomonas cells. A summary of other bacterial strains and plasmids used in this study is provided in Table 2. P. fluorescens and E. coli strains were routinely grown in Luria-Bertani (LB) medium [24] at $28^{\circ} \mathrm{C}$ and $37^{\circ} \mathrm{C}$, respectively. In $\mathrm{P}_{\mathrm{i}}$ limitation studies, $P$. fluorescens strains were grown in a defined citrate medium [25] that contains citric acid $(4.0 \mathrm{~g} / \mathrm{l})$, 
Table 2: Bacterial strains, plasmids and oligonucleotide primers used in this work

\begin{tabular}{|c|c|c|}
\hline Strain, plasmid or primer & Relevant properties & Source/reference \\
\hline \multicolumn{3}{|l|}{ P. fluorescens } \\
\hline SBW25 & Wild type strain isolated from sugar beet & {$[10]$} \\
\hline SBW25-Sm & Spontaneous Smr derivative of SBW 25 & {$[20]$} \\
\hline $\mathrm{SBW} 25 \Delta$ dapB & DAP/lysine auxotroph of SBW 25 & {$[20]$} \\
\hline PIL082 & The hxcR-'dapB'lacZ IVET fusion strain of SBW25 $\Delta$ dapB, Tcr & This work \\
\hline PIL082-I & $\Delta$ dapB DUP(hxcS-psp):::pIVETD, the psp-'dapB'lacZ IVET fusion strain, $\mathrm{Tc}^{r}$ & This work \\
\hline SBW25-lacZ & SBW25 marked with 'lacZ in a phage locus & {$[28]$} \\
\hline PBR826 & $\Delta p s p$ or $\Delta p$ flu2427, SBW25 with a nonpolar deletion of $p s p, T^{s}$ & This work \\
\hline PBR827 & $\Delta p s t C$ or $\Delta p$ flu3317, SBW25 with a nonpolar deletion of pstC, $T^{s}$ & This work \\
\hline PBR828 & $\Delta h x c R$ or $\Delta p$ flu2424, SBW25 with a nonpolar deletion of $h x c R, T c^{s}$ & This work \\
\hline PBR829 & DUP(hxcS-psp)::pUIC3, the psp-'lacZ fusion strain of SBW25, Tcr & This work \\
\hline PBR830 & DUP(pstS-pstC)::pUIC3, the pstC-'lacZ fusion strain of SBW25, Tcr & This work \\
\hline \multicolumn{3}{|l|}{ Plasmid } \\
\hline PRK2013 & Helper plasmid, $\mathrm{Tra}^{+}, \mathrm{Km}^{\mathrm{r}}$ & [34] \\
\hline pUIC3 & Integration vector with promoterless 'lacZ, oriR6K, $\mathrm{Mob}^{+}, \mathrm{Tc}^{\mathrm{r}}$ & [21] \\
\hline PIVETD & DAP-based IVET vector, pUIC3 with promoterless 'dapB'lacZ, oriR6K, Tcr & {$[20]$} \\
\hline PIVETD-13 & pIVETD containing $0.8 \mathrm{~kb}$ psp fragment fused to 'dapB'lacZ, oriR6K, $T \mathrm{c}^{\mathrm{r}}$ & This work \\
\hline pUIC3-6I & pUIC3 carrying psp-'lacZ fusion, oriR6K, $\mathrm{Tc}^{r}$ & This work \\
\hline PUIC3-62 & pUIC3 carrying pstC-'lacZ fusion, oriR6K, $\mathrm{Tc}^{\mathrm{r}}$ & This work \\
\hline PUIC3-4I & pUIC3 containing $1.6 \mathrm{~kb}$ psp deletion fragment, oriR6K, $\mathrm{Tc}^{\mathrm{r}}$ & This work \\
\hline pUIC3-63 & pUIC3 containing I. $6 \mathrm{~kb}$ pst $C$ deletion fragment, oriR6K, $\mathrm{Tc}^{\mathrm{r}}$ & This work \\
\hline pUIC3-64 & pUIC3 containing $1.9 \mathrm{~kb} h x c R$ deletion fragment, oriR6K, $\mathrm{Tc}^{r}$ & This work \\
\hline \multicolumn{3}{|c|}{ 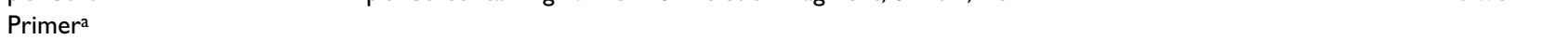 } \\
\hline psp-l & GAAGATCTGTTGCGTGCCCTGGAAG & \\
\hline psp-2 & cagcatgcggatccgttgacggaAGCGAGGGTCATGGATACCG & \\
\hline psp-3 & tccgtcaacggatccgcatgctgCGGCAACACCAACGTCTGC & \\
\hline psp-4 & GAAGATCTGCTGCTGGAAATCCACGGT & \\
\hline hxcR-I & GAGATCTGCGGTAATGGCCACCTATG & \\
\hline$h x c R-2$ & cagcatgcggatccgttgacggaCGCTGCACCTCGCTGATCGA & \\
\hline hxcR-3 & tccgtcaacggatccgcatgctgTCGACGACGATGTGCGCAGC & \\
\hline$h x c R-4$ & GAGATCTATGCCAATTCAAACGCGCCA & \\
\hline pstC-I & GAGATCTTCACCAAGCACCTGGCGG & \\
\hline pstC-2 & cagcatgcggatccgttgacggaAGCCGTGCTTTTCCATGCCT & \\
\hline pstC-3 & tccgtcaacggatccgcatgctgTCGCTGTTTGCACCGGCCAAC & \\
\hline pstC-4 & GAGATCTGCCGGTGGGCAAGACGATTTTC & \\
\hline
\end{tabular}

a Restriction sites incorporated into the primers are underlined. Complementary sequence designed for the SOE-PCR is indicated by small letters.

$\left(\mathrm{NH}_{4}\right)_{2} \mathrm{SO}_{4}(1.0 \mathrm{~g} / \mathrm{l})$ and $\mathrm{MgSO}_{4} \cdot 7 \mathrm{H}_{2} \mathrm{O}(0.2 \mathrm{~g} / \mathrm{l})$. Additionally, $\mathrm{Na}_{2} \mathrm{HPO}_{4}$ and $\mathrm{KH}_{2} \mathrm{PO}_{4}$ were added at the concentration of $6.0 \mathrm{~g} / \mathrm{l}$ and $3.0 \mathrm{~g} / \mathrm{l}$, respectively in phosphate rich medium (PR), whereas in phosphate-limited (PL) medium they are added at $2.4 \mathrm{mg} / \mathrm{l}$ and $1.2 \mathrm{mg} / \mathrm{l}$, respectively. IVET fusion strains of SBW25 $\triangle$ dapB were cultivated in minimal M9 medium [24] supplemented with diaminopimelate (DAP) and lysine at the concentration of 800 $\mu \mathrm{g} / \mathrm{ml}$ and $60 \mu \mathrm{g} / \mathrm{ml}$, respectively. A Pseudomonas selective CFC (Cetrimide, Fucidin, and Cephalosporin) from Oxoid (Hampshire, UK) was supplemented in the LB agar plates to select for $P$. fluorescens recovered from plant. When necessary, antibiotics were added to the following concentrations $(\mu \mathrm{g} / \mathrm{ml})$ : tetracycline $(\mathrm{Tc}), 15$; kanamycin $(\mathrm{Km}), 50$; Streptomycin $(\mathrm{Sm}), 100$; nitrofurantoin $(\mathrm{NF})$, 100. Nutritional supplements of diaminopimelate (DAP) and lysine were added at the final concentration of 800 $\mu \mathrm{g} / \mathrm{ml}$ and $60 \mu \mathrm{g} / \mathrm{ml}$, respectively.

\section{DNA manipulations}

General DNA recombination techniques were used according to standard protocols [24]. Plasmids and DNA fragments from agarose gels were extracted and purified using kits from Qiagen (Biolab Ltd., Auckland, NZ). DNA restriction and modification enzymes and T4 DNA ligase were purchased from Roche (Auckland, NZ) and used as recommended by the manufacturer. $P$. fluorescens SBW25 genes were amplified from genomic DNA by polymerase chain reactions (PCR) using Taq DNA polymerase from Invitrogen (Auckland, NZ). DNA was sequenced using the BigDye Terminator Sequencing kit (Applied Biosystems, Auckland, NZ) on an automated DNA Sequencer, model 310 (Perkin Elmer). 


\section{Construction of lacZ fusions and assays for $\beta$ galactosidase activity}

To construct $l a c Z$ fusions to $p s p$ and $p s t C$, DNA fragments ( 800 bp) were amplified from P. fluorescens SBW25 by using primer pairs "psp-1/-2" and "pstC-1/-2", respectively. The PCR products were first cloned into pCR8/GW/ TOPO (Invitrogen, Auckland, NZ) and sequence identity was confirmed by DNA sequencing. The DNA was retrieved by BglII and BamHI digestion and cloned into the BglII site of pUIC3 (Mob+, Tra'). Gene orientation was then determined by restriction analysis of BglII and EcoRI. The resulting plasmid (pUIC3-61 or pUIC3-62) was mobilized into $P$. fluorescens SBW25 by a general procedure of plasmid conjugation with the help of pRK2013 $\left(\mathrm{Mob}^{+}, \mathrm{Tra}^{+}\right)$. Transconjugants were selected on LB plates supplemented with nitrofurantoin (to counter-select $E$. coli) and Tc. The recombinant plasmid integrated into the genome by a single event of insertion-duplication. Correct integration was confirmed by PCR.

$\beta$-galactosidase activities were assayed by using 4 MUG (4methylumbelliferyl- $\beta$-D-galactoside) as the enzymatic substrate. The product (7-hydroxy-4-methylcoumarin, 4 MU) was detected using a Hoefer DyNA Quant 200 fluorometer (Pharmacia Biotech, Auckland, NZ) with an emission and excitation wavelength of $460 \mathrm{nM}$ and $365 \mathrm{nM}$, respectively. Cell density was determined by measuring the absorbance of the culture at $600 \mathrm{~nm}$. The enzyme activity was expressed as "amol $4 \mathrm{MU} / \mathrm{min} / \mathrm{cell} "$.

\section{Site-directed mutagenesis of P. fluorescens SBW25 and fitness assays}

The $p s p$, pst $C$ and $h x c R$ deletion mutants were constructed by a previously described protocol of SOE-PCR (splicing by overlapping extension using the polymerase chain reaction) [26] in conjunction with a two-step allelic exchange strategy. Two DNA fragments flanking the deleted region of a gene were amplified by two primer pairs, e.g. psp-1/psp-2 and psp-3/psp- 4 in the case of $p s p$ deletion. The two DNA fragments were then joined together by PCR reaction using primers psp- 1 and psp- 4 (this was possible because of the complementary sequences incorporated into primers psp-2 and psp-3). The resulting $\sim 1.6 \mathrm{~kb}$ DNA fragment was cloned into pCR8/GW/TOPO and sequenced to ensure that it was error-free. The DNA fragment was retrieved by BglII then cloned into the delivery vector pUIC3 to generate pUIC341 (for $\Delta p s p$ ), pUIC3-63 (for $\Delta p s t C$ ) and pUIC3-64 (for $\Delta h x c R)$.

To delete $p s p$ (or $p s t C$ or $h x c R$ ) from the genome of SBW25, pUIC3-41 (or pUIC3-63 or pUIC3-64) was mobilized into SBW25 by conjugation with the help of pRK2013 $\left(\mathrm{Tra}^{+}\right)$. Integration by homologous recombination was selected on LB plates supplemented with nitrofurantoin, tetracycline and X-gal. To select for allelic exchange mutants, purified single blue-colored transconjugants were subjected to cycloserine enrichment as previously described [27]. Allelic exchange mutants (white-colored and Tc-sensitive) were examined by PCR to distinguish the mutants from wild type.

Fitness of the mutant strains in laboratory media and in planta was assessed by direct competition with a lacZmarked "wild-type" strain of $P$. fluorescens SBW25 (SBW25-lacZ) as previously described [28]. The mutant and the wild-type competitor were counted on LB agar plates supplemented with X-gal. Population densities $\left(N_{i}\right)$ determined at time $t=0$ and at $t=T$ were used to calculated the Malthusian parameter [29], which is the average rate of increase and was calculated for both competitors: $m_{i}=\ln \left[N_{i}(T) / N_{i}(0)\right]$. Relative fitness is expressed here as the selection rate constant (SRC): $r_{i j}=m_{i}-m_{j}$, resulting in a fitness of zero when competing organisms are equally fit.

\section{In vivo expression technology analysis and plant experiments}

Isolation process of the PIL082 fusion was previously described [20]. To construct the $p s p$ IVET fusion, the $\sim 800$ bp DNA fragment used for the construction of $p s p-l a c Z$ fusion (pUIC3-61) was cloned into the BglII site of the DAP-based IVET vector PIVETD to generate pIVETD-13, which has $p s p$ fused to the promoterless ' $d a p B$ reporter. PIVETD-13 was then integrated into the $p s p$ locus of SBW25 by conjugation with the help of pRK2013. The obtained $p s p$ IVET fusion strain was named PIL082-1.

Ability of the IVET strains to grow in planta was assessed by a competitive colonization assay in sugar beet seedlings [22]. The fusion strain was mixed 1:1 with a competitor strain (SBW25-Sm) and inoculated onto coated sugar beet (Beta vulgaris var. Amethyst) seeds, which were germinated and cultivated in $15 \mathrm{ml}$ plastic tubes filled with non-sterile vermiculite. About 1000 cells were inoculated and two weeks after plant sowing bacteria from the shoot and rhizosphere (roots with attached vermiculite) were counted on LB plates supplemented with lysine, DAP, CFC and X-gal. The competitor strain SBW25-Sm was counted in LB plates with CFC and streptomycin and present in all treatments at the similar levels $\left(\sim 10^{4}\right.$ per shoot and $\sim 10^{6}$ per rhizosphere).

\section{Immunochemical analysis}

Two antisera were used in these experiments. A rabbit antiserum to a conjugated, synthetic peptide corresponding to the N-terminus of the human DING protein, was prepared and used as previously described [3]. The second antiserum (anti-Psp) was made in NZ White rabbits, with recombinant DING protein from $P$. fluorescens SBW25 [9] 
as the antigen, using standard methods. Double immunodiffusion was carried out in $1.5 \%(\mathrm{w} / \mathrm{v})$ agarose gels. Western blotting was carried out as previously described [3], using 12\% precast SDS-PAGE gels (Bio-Rad, Auckland, NZ). The wild-type SBW25 and mutant strains $\Delta p s p$ and $\Delta h x c R$ were grown to the same cell density in $5 \mathrm{ml}$ cultures. Supernatants were freeze-dried, and resuspended in $0.1 \mathrm{ml} 30 \mathrm{mM}$ Tris-HCl buffer ( $\mathrm{pH} 7.5$ ). Cell pellets were similarly resuspended, sonicated and re-centrifuged. For immunodiffusion, $0.025 \mathrm{ml}$ samples were used, and for electrophoresis, $0.015 \mathrm{ml}$, in each case. Triplicate cultures were analyzed, and a typical result is shown in each case.

\section{Computational analysis}

PstS was identified by BLAST (basic local alignment search tool) search of the complete SBW25 genome sequence using the deduced amino acid sequence of $p s p$ ( $p f l u 2427$ ). The SBW25 Psp and PstS sequences were then used in BLAST searches for homologues in other Pseudomonas species or strains deposited in the Pseudomonas genome database v2 [30]. Amino acid sequences were aligned using ClustalX program [31] and a phylogenetic tree constructed using the neighbor-joining method [32]. The tree was displayed in TreeView [33].

\section{Abbreviations}

$\mathrm{P}_{\mathrm{i}}$, inorganic phosphate; IVET, In vivo expression technique; DAP, diaminopimelic acid; SRC, selection rate constant; EST, expressed sequence tag; $\mathrm{P}_{\mathrm{i}}$, inorganic phosphate; LB, Luria-Bertani; PR, phosphate rich; PL, phosphate limited; CFC, cetrimide, fucidin, and cephalosporin; NF, nitrofurantoin; Tc, tetracycline; Km, kanamycin; Sm, streptomycin. PCR, polymerase chain reactions. $4 \mathrm{MU}$, 7-hydroxy-4-methylcoumarin.

\section{Authors' contributions}

XXZ, KS and PBR designed the experiments and interpreted the results. XXZ performed the genetic manipulations, enzymatic assays and plant experiments and drafted the manuscript. KS conducted the immunochemical experiments, and contributed to the manuscript. RM was involved in the genetic and immunochemical studies. All authors read and approved the final manuscript.

\section{Acknowledgements}

We thank Stephen Giddens for maintaining the SBW25 IVET database. KS thanks the Staff Research Fund of the University of Auckland Research Committee for financial support.

\section{References}

I. Hain NA, Stuhlmuller B, Hahn GR, Kalden JR, Deutzmann R, Burmester GR: Biochemical characterization and microsequencing of a 205-kDa synovial protein stimulatory for $T$ cells and reactive with rheumatoid factor containing sera. I Immunol 1996, 157:1773-1780.

2. Kumar V, Yu S, Farell G, Toback FG, Lieske JC: Renal epithelial cells constitutively produce a protein that blocks adhesion of crystals to their surface. Am J Physiol Renal Physiol 2004, 287:373-383.

3. Adams L, Davey S, Scott K: The DING protein: an autocrine growth-stimulatory protein related to the human synovial stimulatory protein. Biochim Biophys Acta 2002, 1 586:254-264.

4. Belenky M, Prasain J, Kim H, Barnes S: DING, a genistein target in human breast cancer: a protein without a gene. J Nutr 2003, I33(7 Suppl):2497S-250IS.

5. Berna A, Bernier F, Scott K, Stuhlmuller B: Ring up the curtain on DING proteins. FEBS Lett 2002, 524:6-10.

6. Morales R, Berna A, Carpentier P, Contreras-Martel C, Renault F, Nicodeme M, Chesne-Seck ML, Bernier F, Dupuy J, Schaeffer C, Diemer H, Van-Dorsselaer A, Fontecilla-Camps JC, Masson P, Rochu D, Chabriere E: Serendipitous discovery and X-ray structure of a human phosphate binding apolipoprotein. Structure 2006, I4:60|-609.

7. Wanner BL: Gene regulation by phosphate in enteric bacteria. J Cell Biochem 1993, 5 I:47-54.

8. Berna A, Bernier F, Chabriere E, Perera T, Scott K: DING proteins; novel members of a prokaryotic phosphate-binding protein superfamily which extends into the eukaryotic kingdom. Int J Biochem Cell Biol 2008, 40: I70-I75.

9. Scott K, Wu L: Functional properties of a recombinant bacterial DING protein: comparison with a homologous human protein. Biochim Biophys Acta 2005, 1744:234-244.

10. Bailey MJ, Lilley AK, Thompson IP, Rainey PB, Ellis RJ: Site directed chromosomal marking of a fluorescent pseudomonad isolated from the phytosphere of sugar beet; stability and potential for marker gene transfer. Mol Ecol 1995, 4:755-763.

II. Ahn S, Moniot S, Elias M, Chabrière E, Kim D, Scott K: Structurefunction relationships in a bacterial DING protein. FEBS Lett 2007, 58 I:3455-3460.

12. Luecke $H$, Quiocho FA: High specificity of a phosphate transport protein determined by hydrogen bonds. Nature 1990, 347:402-406.

13. Inatsuka CS, Julio SM, Cotter PA: Bordetella filamentous hemagglutinin plays a critical role in immunomodulation, suggesting a mechanism for host specificity. Proc Natl Acad Sci USA 2005, 102: 18578-18583.

14. Ball G, Durand E, Lazdunski A, Filloux A: A novel type II secretion system in Pseudomonas aeruginosa. Mol Microbiol 2002, 43:475-485.

I5. Sandkvist M: Biology of type II secretion. Mol Microbiol 200I, 40:27I-283.

16. The SignalP 3.0 Server [http://www.cbs.dtu.dk/services/SignalP/]

17. Tan AS, Worobec EA: Isolation and characterization of two immunochemically distinct alkaline phosphatases from Pseudomonas aeruginosa. FEMS Microbiol Lett 1993, 106:28I-286.

18. Nikata T, Sakai Y, Shibat K, Kato J, Kuroda A, Ohtake H: Molecular analysis of the phosphate-specific transport (pst) operon of Pseudomonas aeruginosa. Mol Gen Genet 1996, 250:692-698.

19. Monds RD, Newell PD, Schwartzman JA, O'Toole GA: Conservation of the Pho regulon in Pseudomonas fluorescens PfO-I. Appl Environ Microbiol 2006, 72:1910-1924.

20. Gal M, Preston GM, Massey RC, Spiers AJ, Rainey PB: Genes encoding a cellulosic polymer contribute toward the ecological success of Pseudomonas fluorescens SBW25 on plant surfaces. $\mathrm{Mol}$ Ecol 2003, I 2:3 109-3 I2I.

21. Rainey PB: Adaptation of Pseudomonas fluorescens to the plant rhizosphere. Environ Microbiol 1999, 1:243-257.

22. Zhang X-X, Rainey PB: The role of a PI-type ATPase from Pseudomonas fluorescens SBW25 in copper homeostasis and plant colonization. Mol Plant-Microbe Interact 2007, 20:58I-588.

23. Hanahan D: Studies on transformation of Escherichia coli with plasmids. J Mol Biol 1983, 166:557-580.

24. Sambrook J, Russel DW: Molecular cloning - a laboratory manual. 3rd edition. Cold Spring Harbor, New York: Cold Spring Harbor Laboratory Press; 200I.

25. Al-Aoukaty A, Appanna VD, Huang J: Exocellular and intracellular accumulation of lead in Pseudomonas fluorescens ATCC I3525 is mediated by the phosphate content of the growth medium. FEMS Microbiol Lett 199 I, 67:283-290.

26. Horton RM, Hunt HD, Ho SN, Pullen JK, Pease LR: Engineering hybrid genes without the use of restriction enzymes: gene splicing by overlap extension. Gene 1989, 77:61-68. 
27. Zhang $X-X$, Rainey PB: Genetic analysis of the histidine utilization (hut) genes in Pseudomonas fluorescens SBW25. Genetics 2007, 176:2165-2176.

28. Zhang $X-X$, Rainey PB: Construction and validation of a neutrally-marked strain of Pseudomonas fluorescens SBW25. J Microbiol Methods 2007, 71:78-8I.

29. Lenski RE: Quantifying fitness and gene stability in microorganisms. Biotechnology 1991, 15:173-192.

30. The Pseudomonas Genome Database v2 [http://www.pseu domonas.com/]

31. Thompson JD, Gibson TJ, Plewniak F, Jeanmougin F, Higgins DG: The ClusterX windows interface: flexible strategies for multiple sequence alignment aided by quality analysis tools. Nucleic Acids Res 1997, 25:4876-4882.

32. Saitou N, Nei M: The neighbor-joining method: a new method for reconstructing phylogenetic trees. Mol Biol Evol 1987, 4(4):406-425.

33. Page RDM: TreeView: an application to display phylogenetic trees on personal computers. Comp Appl Biosci 1996, I 2:357-358.

34. Ditta G, Stanfield S, Corbin D, Helinski DR: Broad host range DNA cloning system for Gram negative bacteria: construction of a gene bank of Rhizobium meliloti. Proc Natl Acad Sci USA |980, 77:7347-735|

Publish with Biomed Central and every scientist can read your work free of charge

"BioMed Central will be the most significant development for disseminating the results of biomedical research in our lifetime. "

Sir Paul Nurse, Cancer Research UK

Your research papers will be:

- available free of charge to the entire biomedical community

- peer reviewed and published immediately upon acceptance

- cited in PubMed and archived on PubMed Central

- yours - you keep the copyright

Submit your manuscript here:

http://www.biomedcentral.com/info/publishing_adv.asp
BiolMedcentral 